\title{
L'influence du positivisme et de l'inductivisme dans les manuels de sciences physiques de la première moitié du XXe siècle
}

Jean-Michel Dusseau et Pierre Fréchengues

\section{OpenEdition}

Journals

Édition électronique

URL : http://journals.openedition.org/trema/1811

DOI : 10.4000/trema.1811

ISSN : 2107-0997

Éditeur

Faculté d'Éducation de l'université de Montpellier

\section{Édition imprimée}

Date de publication : 1 décembre 1998

Pagination : 51-67

ISSN : 1167-315X

\section{Référence électronique}

Jean-Michel Dusseau et Pierre Fréchengues, «L'influence du positivisme et de l'inductivisme dans les manuels de sciences physiques de la première moitié du XXe siècle», Tréma [En ligne], 14 | 1998, mis en ligne le 01 décembre 1998, consulté le 01 mai 2019. URL : http://journals.openedition.org/ trema/1811 ; DOI : 10.4000/trema.1811

Ce document a été généré automatiquement le 1 mai 2019.

Trema 


\title{
L'influence du positivisme et de l'inductivisme dans les manuels de sciences physiques de la première moitié du XXe siècle
}

\author{
Jean-Michel Dusseau et Pierre Fréchengues
}

\section{Introduction}

1 L'histoire de l'introduction d'un concept de physique dans l'enseignement ne peut être coupée de l'histoire de l'émergence de ce concept dans la communauté scientifique. Dans cet article nous ne rappellerons pas les détails de l'émergence de l'électron (Fréchengues \& Dusseau, 1998) mais nous essaierons de re-situer l'introduction de ce dernier dans les manuels d'enseignement compte tenu des choix épistémologiques qui prévalaient alors chez les auteurs.

2 Le terme d'électron représente pour C. Johnstone Stoney, qui le propose en 1891, l'unité naturelle de charge électrique, celle que porte un ion monovalent dans l'électrolyse. Cette idée qu'il existe une charge élémentaire d'électricité, en germe à la fin du siècle dernier chez les théoriciens de l'électromagnétisme comme FitzGerald, Larmor ou Lorentz, se concrétise à travers un certain nombre d'expériences sur les rayons cathodiques. Tout d'abord, en 1895, Perrin montre que ces rayons n'ont rien d'ondulatoire car constitués de particules chargées. Puis dans une publication datée du 30 avril 1897, J.J. Thomson précise que les rayons cathodiques sont composés de particules environ mille fois plus petites que l'atome d'hydrogène et détermine le rapport $\mathrm{e} / \mathrm{m}$ de ces particules, qu'il dénomme « corpuscules » et qu'il refusera jusque vers 1915 d'appeler des électrons. En effet, considérant l'atome comme sécable, ces corpuscules constituent selon lui les «atomes primordiaux ", bases des atomes chimiques ordinaires, tandis que les électrons sont 
souvent considérés à cette époque (Lelong, 1897) comme des singularités de l'éther, c'està-dire des charges électriques indépendantes de la matière.

Or, même si l'électron apparait à partir des années 1910 dans quelques rares manuels scolaires comme permettant d'expliquer les rayons cathodiques alors mentionnés dans les programmes de physique de Terminale, l'introduction explicite de l'électron dans ces programmes n'aura lieu qu'en 1947. Nous montrons que ce décalage n'est pas dû à la non diffusion du concept depuis le cercle de la recherche vers celui de l'enseignement, mais plutôt à son inutilité dans le cadre du positivisme et de l'inductivisme alors prégnants.

POUILLET M. :Notions Générales de Physique et de Météorologie à l'Usage de la Jeunesse. Paris, Béchet Jeune, 1850.

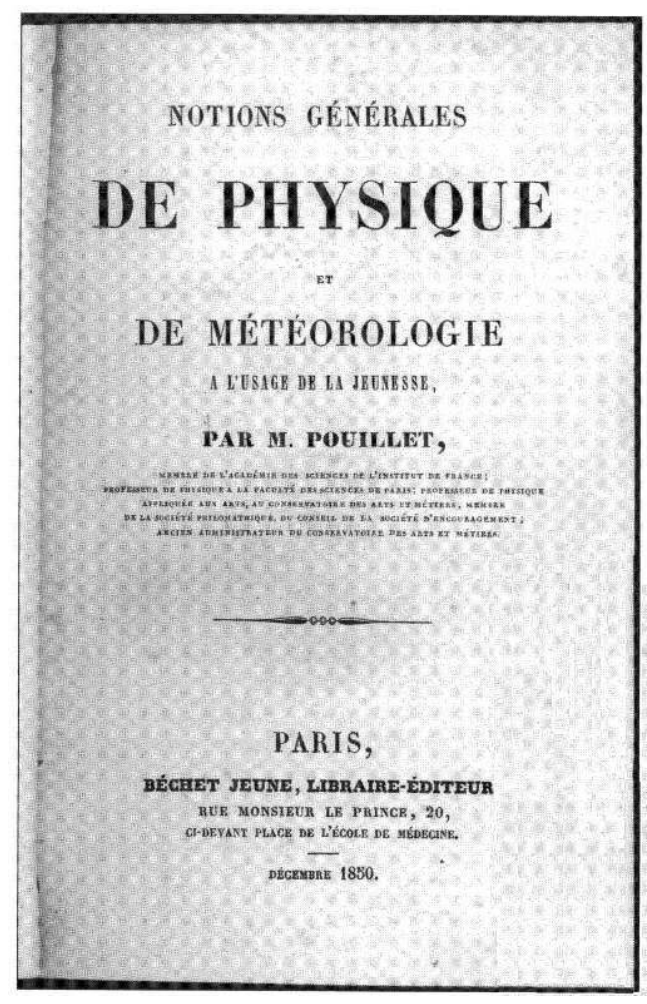


LEMOINE J. et VINCENT G. : Cours Élémentaire de Physique (Classe de Mathématiques). Paris, Librairie Classique Eugène Belin, 1931.

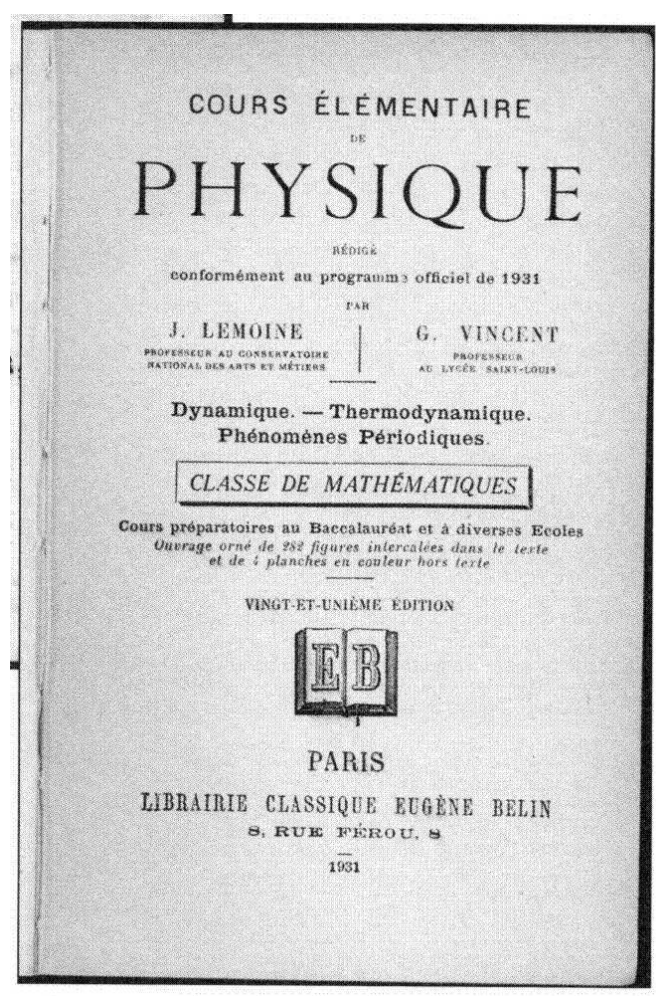

4 On peut remarquer que, jusqu'à la veille de 1914 , le positivisme va être un frein à l'acceptation de la discontinuité (de la matière, de l'électricité...) par une grande partie de la communauté scientifique. Cette absence de consensus fort sur la discontinuité va laisser de nombreux auteurs de manuels dans une prudente expectative pendant longtemps. De plus, l'inductivisme préconisé comme méthode pédagogique naturelle, en concomitance avec l'expérimentation en classe, va rendre très difficile l'introduction de l'atomisme et de l'électron.

$5 \quad$ Nous avons privilégié deux séries d'ouvrages scolaires destinés aux classes de Première et Terminale et édités pendant une période allant bien au-delà de l'installation du concept dans la communauté scientifique :

- celle dont un des auteurs est Faivre-Dupaigre, parce qu'elle couvre de 1905 (date de la collaboration de Faivre-Dupaigre - qui a été de 1910 à 1921 président du jury de l'agrégation de sciences physiques - comme simple professeur avec Fernet) à 1946 où, comme inspecteur général honoraire, Faivre-Dupaigre publie encore des manuels avec Lamirand et Joyal ;

- celle de Lemoine et Vincent, dont les rééditions successives (caractéristiques d'une diffusion importante) de leur cours élémentaire de physique couvrent la période 1908-1931.

Bien entendu, il était intéressant de mettre en parallèle à ces collections d'un côté quelques ouvrages de l'enseignement supérieur et d'un autre côté des manuels à destination de l'enseignement primaire supérieur (cycle court visant à former des cadres moyens). 


\section{Nature de l'électricité et discontinuité de la matière dans les ouvrages de sciences physiques.}

\section{1. Ouvrages généraux et manuels de 1850 à 1914 : le règne du positivisme}

7 À la fin du siècle dernier et jusque vers 1914 les ouvrages à destination des élèves ou du grand public s'intéressent peu à la nature de l'électricité. D'ailleurs ils ne s'interrogent pas davantage sur la constitution de la matière. Berthelot maintient la censure de l'atomistique que Dumas (1837) avait imposée avant lui dans l'enseignement français, même si des savants comme Wurtz (1879) ont grandement contribué au développement de la théorie atomique.

Dans son ouvrage Notions générales de Physique et de météorologie à l'usage de la jeunesse paru en 1850, Pouillet demande (p. 281) : «Le mouvement de l'électricité est-il un mouvement de translation ou un mouvement de vibration?", et il conclut après avoir cité un certain nombre d'expériences, qu'il reste beaucoup de recherches à faire pour se rendre compte de toutes les conditions et de tous les éléments de son mouvement de vibration.

De même Rambosson dans La science populaire ou revue des connaissances et de leurs applications aux arts et à l'industrie paru en 1863 indique (p. 126) :

"L'espèce d'électricité que l'on communique à un corps dépend non seulement du corps frottant, mais aussi de l'état de surface du corps frotté. On peut, par exemple, donner à une tige même de verre les deux électricités (vitreuse et résineuse) à la fois; il suffit pour cela qu'elle soit polie par l'une de ses extrémités et dépolie par l'autre. Naturellement, on est porté à se demander pourquoi dans tel cas c'est l'une des électricités qui se développe plutôt que l'autre ; mais la science n'est pas encore assez avancée pour résoudre cette question. »

10 En 1867, Daguin publie le troisième tome, consacré à l'électricité, de son Traité de Physique. Il présente de façon assez détaillée les théories de Franklin et de Symmer, avouant une préférence pour cette dernière car (p. 103) :

" on doit la considérer comme formant un jalon sur le chemin qui doit conduire plus tard à la découverte de la cause première; il faudra un jour renoncer à l'idée des fluides, mais le mode d'action de la cause réelle devra être, sans doute, toujours interprété de la même manière, car les choses se passent comme s'ils existaient.

" Aujourd'hui, on cherche à rattacher les actions électriques à des mouvements particuliers de l'éther, et à rapprocher ainsi les phénomènes de l'électricité, de ceux de la chaleur et de la lumière. Nous verrons, en effet, qu'il y a de nombreux points de contact entre ces divers ordres de phénomènes; mais la science, à ce sujet, ne présente encore, pour ainsi dire que des pressentiments, et l'on n'est pas encore parvenu à lier par cette nouvelle théorie l'ensemble des phénomènes électriques. »

11 Ce n'est qu'à la fin de la présentation de ses Recherches sur l'Électricité publié en 1883 que Planté consacre quelques pages aux «Analogies entre les phénomènes électriques et les effets produits par des actions mécaniques. Conséquences relatives à la nature de l'électricité.» Il termine ainsi (p. 314) :

«Pour résumer en quelques mots les vues exposées ci-dessus, nous pensons que l'électricité peut-être considérée comme un mouvement de la matière pondérable - mouvement de transport d'une très petite masse de matière animée d'une très grande vitesse, s'il s'agit de la décharge électrique - et mouvement vibratoire très rapide des molécules de la matière, s'il s'agit de sa transmission à distance sous la formedynamique ou de sa manifestation sous la forme statique à la surface des corps. » 
Pile au bichromate

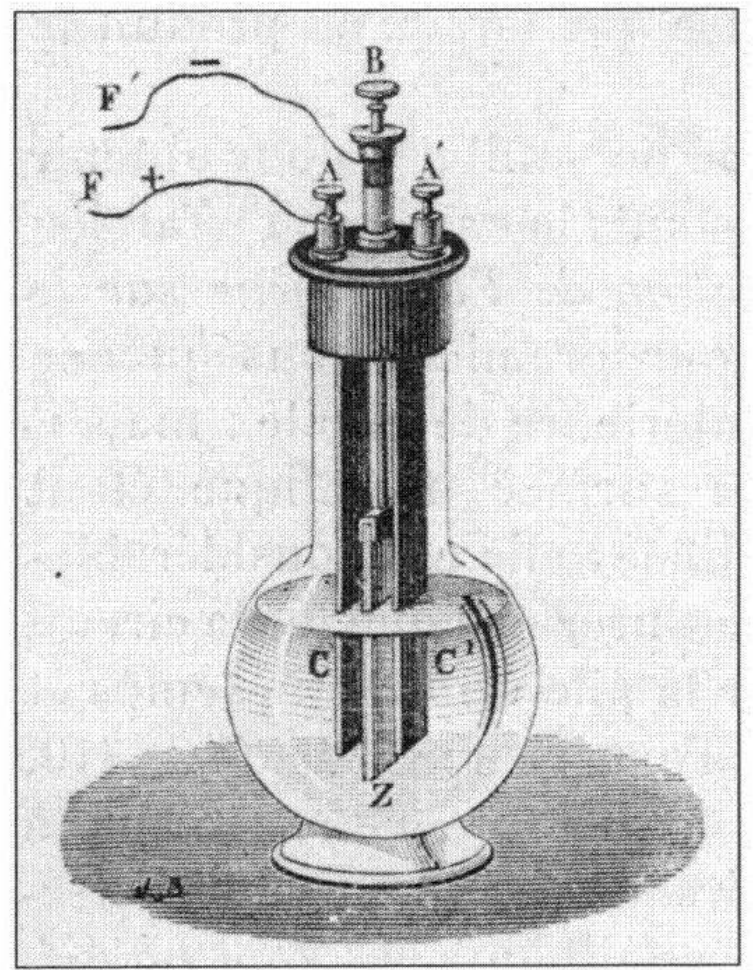

Galvanomètre de Nobili

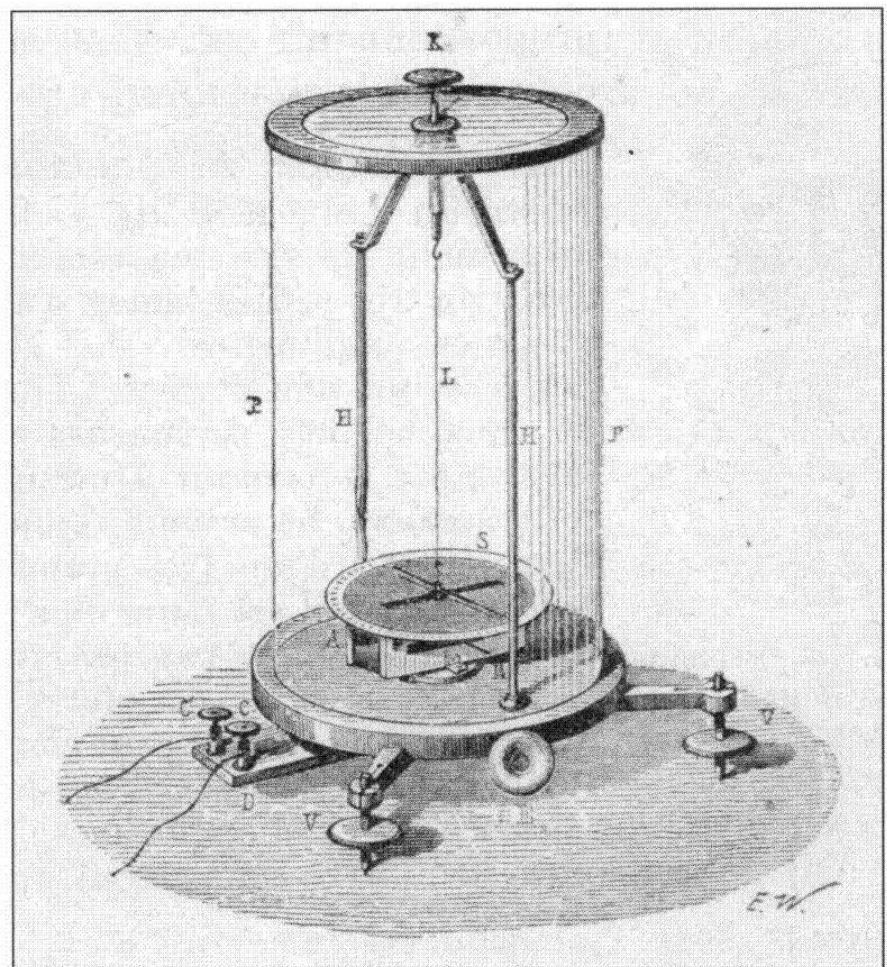

(2 figures précédentes) : MÉTRAL P. : Cours de Physique pour l'Enseignement Primaire Supérieur. Paris Masson et Cie Éditeurs, 1907. (nouvelle édition), Ch. 8, p. 292, fig. 328 \& Ch. 10, p. 319, fig. 349 
Quant à Pécheux dans son Précis de Physique Industrielle de 1899, il affirme (p. 380) :

«Il n'y a qu'une seule espèce d'électricité, L'électricité étant une forme spéciale de l'énergie, se manifestant par divers phénomènes ayant entre eux une grande analogie, il n'y a pas et il ne peut y avoir deux sortes d'électricité. Les désignations toujours admises d'électricité positive et d'électricité négative ne peuvent servir qu'à indiquer deux modes d'électrisation différents, mais non deux espèces différentes d'électricité. "

13 Ce à quoi les auteurs préfèrent s'atteler, c'est à une description phénoménologique et mathématique des phénomènes électriques et de leurs applications. Un des ouvrages le plus achevé dans ce sens est celui de Fernet : Précis de Physique, dont la $28^{\mathrm{e}}$ édition (1905) passe en revue sur plus de 100 pages denses tous les phénomènes connus à l'époque (du pouvoir des pointes aux rayons cathodiques et aux rayons $\mathrm{X}$ ou à la télégraphie sans fil) en ne s'interrogeant jamais sur la nature physique de l'électricité. Il pose simplement (p. 253) : "Par définition, la quantité d'électricité ou masse électrique d'un élément électrisé est considérée comme proportionnelle à la force qu'il exerce, à une distance déterminée, sur un autre élément dont la charge reste constante ».

14 Ce sera encore le cas en 1913, dans un ouvrage, conforme au programme de 1912, publié sous sa direction par Faivre-Dupaigre et Carimey et destiné aux élèves des classes de Première $\mathrm{C}$ et $\mathrm{D}$.

15 Ainsi, dans les ouvrages d'électricité, ou dans les parties correspondantes des manuels scolaires, la question de la nature du courant électrique n'est que très peu abordée. C'est la description minutieuse de phénomènes, d'appareils, ou la présentation de quelques lois (Ohm, Joule,...) qui est privilégiée.

16 L'explication de cette attitude, caractéristique du positivisme ambiant, se trouve dans l'ouvrage de Métral (1907) Cours de Physique pour l'Enseignement Primaire Supérieur, lorsqu'il propose un paragraphe "Hypothèses sur la nature de l'électricité " dans lequel il indique (p. 228) :

"Bien des hypothèses ont été faites sur la nature de l'électricité ; la plus répandue est celle des deux fluides, due à Symmer, dans laquelle on admet l'existence de deux fluides impondérables : le fluide positif et le fluide négatif; ces fluides sont susceptibles de s'associer pour en faire un troisième, le fluide neutre. Celui-ci existe, en quantité indéfinie, sur les corps à l'état neutre, et le frottement aurait pour effet de séparer les deux fluides dont la combinaison forme le fluide neutre. Cette hypothèse est inutile, et il vaut mieux s'en passer. Elle est d'ailleurs, peu vraisemblable. Il est probable que les deux espèces d'électricité, que nous constatons, sont deux effets particuliers d'une même cause que nous ne connaissons pas. On pense, généralement, aujourd'hui, que l'électrisation d'un corps conducteur est une manifestation particulière de l'énergie qui est accompagnée d'une certaine modification du milieu isolant qui l'entoure: cette modification produisant les répulsions et les attractions dont nous avons parlé. Bien que la nature intime de l'électricité nous soit inconnue, nous pourrons étudier et mesurer les phénomènes électriques, et en tirer des applications. En y réfléchissant un peu, on s'aperçoit que cette force physique n'est pas plus mystérieuse que la pesanteur, qui nous paraît plus simple, parce que nous l'observons plus souvent. Quand on constate que la terre attire les corps et qu'elle les fait tomber à sa surface, on n'a pas expliqué la nature de cette attraction, ni la façon dont elle se transmet. La nature intime de la pesanteur nous est encore moins connue que celle de l'électricité. »

Mais dans l'édition correspondant au programme de 1909 il mentionne (p. 292) :

"Quant à la nature des rayons cathodiques, il résulte, de tout ce qui précède, qu'ils sont constitués par des particules matérielles dont le poids serait environ 1/1000 du poids atomique de l'hydrogène; on a été conduit à admettre que, sous l'action de la décharge, les molécules de gaz sont désagrégées d'une façon particulière: les particules qui en résultent sont appelées des ions (gazeux); ces ions, comme ceux de l'électrolyse, sont combinés à une 
certaine charge électrique. La charge électrique d'un ion s'appelle un électron; elle est en quelque sorte, la plus petite quantité d'électricité qui puisse exister, une sorte d'atome électrique. On donne le nom d'ionisation à cette désagrégation des molécules matérielles. Les rayons cathodiques sont produits par les électrons négatifs. ”

Il en est de même dans l'édition de 1908 de l'ouvrage de Lemoine et Vincent, destiné aux classes de mathématiques (p. 433) : "On explique les rayons cathodiques en admettant que la nature gazeuse raréfiée des tubesse trouve, sous l'influence de la décharge électrique, dissociée en fragments très petits électrisés, que l'on appelle les corpuscules, ou les électrons ou d'une façon plus générale, les ions [...] ». On note l'ambiguïté du langage, liée très certainement à l'attitude de J.J. Thomson.

Cette question de composants ultimes de la matière concerne également à la même époque la molécule et l'atome, dont on parle obligatoirement en chimie, mais toujours avec une prudente réserve, comme d'une fiction sans impact sur la réalité.

Ombre produite par une croix placée dans le faisceau cathodique.

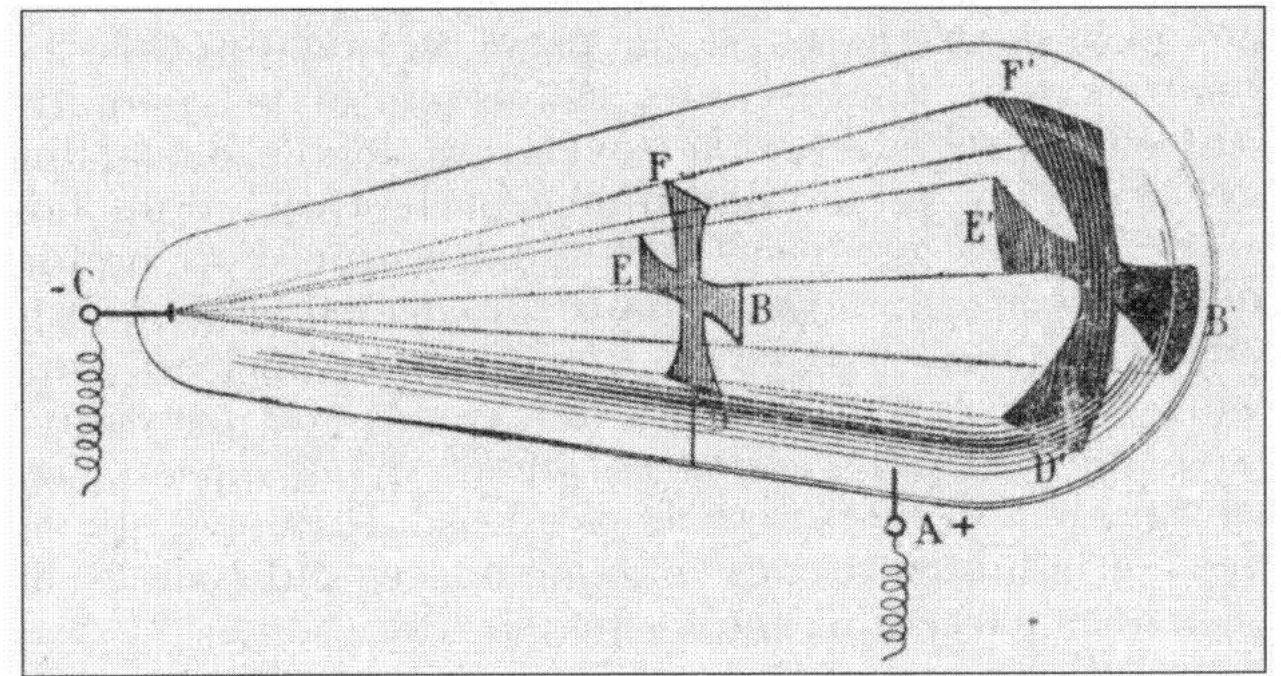

LEMOINE J. \& VINCENT G. : Cours Élémentaire de Physique. Paris, Librairie Classique Eugène Belin Éditeurs, 1931. P. 487, fig. 244.

Si Pécheux dans son ouvrage de 1899 cité précédemment, introduit la molécule comme la particule de matière limite à la divisibilité, Métrai dans son Cours de Chimie de 1905 introduit de manière claire la distinction entre molécule et atome. Mais c'est Drincourt dans ses Trois années de Chimie (en un volume) de 1909 qui ose une comparaison pour le moins hardie (p. 71) :

«Pour rendre saisissable la différence assez subtile entre l'atome et la molécule, on peut emprunter une comparaison à l'ancien régime des bagnes. On sait que, autrefois, les forçats étaient enchaînés deux à deux. L'ensemble des deux forçats pouvait se mouvoir librement, on peut dire que cet ensemble était libre. Mais chacun des deux forçats pris individuellement n'était pas libre; il était captif par rapport à son compagnon de chaîne. L'ensemble libre des deux forçats représente une molécule ; chacun des deux forçats représente un atome. ”

\subsection{Les manuels de sciences physiques de 1914 à 1947}

21 De 1914 à 1924, les programmes et les manuels ne changent pas. C'est peut-être parce que Bruhat dans la $1^{\text {re }}$ édition de son Cours d'électricité à l'usage de l'enseignement supérieur 
scientifique et technique, publié en 1924, consacre le chapitre VII aux bases expérimentales des théories modernes, avec des sous-chapitres sur l'électron, l'atome, les isotopes, les théories électroniques des métaux, les courants dans le vide, les phénomènes photoélectriques et thermoïoniques, la lampe à 3 électrodes, etc., qu'il faut attendre les cours de Bouasse et Brizard publiés en 1925, à destination des élèves de Terminale mathématique pour qu'apparaisse l'électron en tant que grain de matière (p. 347) : «On admet que les molécules des gaz sont dissociées et que ce sont des particules extrêmement petites, les électrons, identiques dans tous les gaz qui interviennent pour former les rayons cathodiques. " Dans leur livre de Première, publié la même année, il n'est pas question d'électron mais d'un certain nombre d'hypothèses, notamment que les corps frottés possèdent un agent appelé électricité. Cependant, si l'on énonce des hypothèses fondamentales elles ne sont qu'une manière de nous représenter les choses (p. 203). "Il ne faut pas exagérer leur signification; les choses se passent comme si ces hypothèses étaient exactes: cela ne veut nullement dire que ces hypothèses représentent la réalité. D'autres hypothèses peuvent tout aussi bien résumer les phénomènes. "

Cependant, la même année (1925), Lemoine et Vincent dans leur Cours élémentaire de Physique à destination des classes de mathématique gardent à l'identique leur explication corpusculaire de 1908 donnée précédemment.

Par contre dans une réédition de leur cours de Première datée aussi de 1925, ils indiquent, de façon surprenante (p. 159) : "Le courant électrique qu'un générateur fait circuler dans une canalisation, qui fait résonner des sonneries, allume des lampes, fait tourner des moteurs, etc., n'est pas constitué par une circulation de matière. Nos sens ne le perçoivent pas comme un courant d'air, et sa nature nous est inconnue. "

En 1927, un livre de Physique couvrant tout le second degré, écrit par Turpain, professeur à la faculté des Sciences de Poitiers, ne mentionne qu'une seule fois l'électron, et ce au détour de la présentation de la lampe triode (p. 956) : « Il [le fonctionnement de la lampe triode] est basé sur l'effet suivant, mis en évidence par Edison en 1883 : un filament incandescent, placé dans le vide, émet des corpuscules négatifs (électrons). »

25 La même année paraissent des ouvrages de chimie dont les auteurs franchissent le pas de l'atomistique. Ainsi Pastouriaux et Cointet s'adressant cependant au public restreint des élèves des écoles normales (p. 31):

"Une théorie célèbre, dite théorie atomique, conduit plus rapidement au même résultat mais elle repose sur des hypothèses relatives à la constitution des corps simples et composés [...]. En conclusion, les deux lois fondamentales de la chimie, loi des proportions définies, loi des proportions multiples, s'interprètent simplement si l'on admet l'hypothèse atomique ".

26 De même Ponsinet indique dans la préface de son traité Principes de l'Électrochimie(p. 1) : "C'est par les phénomènes électrochimiques que s'est révélée l'existence et l'importance des ions, ou particules matérielles chargées d'électricité, et, comme conséquence, ce fait capital que l'électricité, comme la matière, existe sous forme d'éléments distincts, autrement dit sous la forme atomique. " Il présente une théorie électronique de la valence, alors que chez E. Péchard (1927) dans un ouvrage destiné à des étudiants de $1^{\text {re }}$ année d'université, on trouve (p. $31)$ : "Il n'y a pas de règles pour retenir la valence ; à l'usage on apprend facilement la valence des corps simples".

27 En résumé, dans les années 1925-30, l'électron corpusculaire fait une entrée assez timide dans certains manuels dans le cadre de phénomènes comme les rayons cathodiques, ou sous forme de remarques non essentielles, mais jamais en tant qu'élément explicatif de la nature de l'électricité ou en préalable à l'étude des propriétés de cette dernière. Quant à 
la théorie atomique, ses adversaires ont rendu les armes (comme Otswald en 1909) ou sont morts (comme Berthellot en 1907 ou Duhem en 1916). Va-t-elle pour autant apparaitre dans les manuels de chimie? Pas vraiment car ces manuels restent essentiellement descriptifs des propriétés de composés minéraux ou organiques.

En 1931, on peut penser que la cause de l'électron est entendue, puisque, par exemple, dans la nouvelle édition de leur ouvrage à destination des classes de mathématiques, Lemoine et Vincent consacrent plusieurs pages aux électrons et à la structure planétaire de l'atome. Ils appellent la charge de 1,6 x $10^{-19}$ Coulombs le grain d'électricité ou atome d'électricité, et électron le grain d'électricité négative sans support matériel tout en donnant la valeur de la masse de cet électron.

Il est vrai que le Cours de Physique générale du Professeur Ollivier publié en 1932 et destiné aux étudiants des facultés des Sciences en est à présenter l'effet Compton, la mécanique ondulatoire de De Broglie, la diffraction des électrons réalisée par Davisson et Germer, les lampes thermoïoniques et l'oscillographe cathodique.

Pourtant la même année (1932) Faivre-Dupaigre, Lamirand et Brizard dans leur Cours de Physique pour les mathématiques spéciales considèrent (p. 199) « un courant électrique dans un conducteur comme un déplacement de masses d'électricité statique dans ce conducteur. " Le dernier paragraphe de leur chapitre sur l'Électrodynamique est intitulé (p. 202) : Nouvelle conception de la quantité d'électricité. On peut y lire :

"Les deux définitions qui viennent d'être données de l'intensité d'un courant, la définition électrochimique et la définition électromagnétique, envisagent simplement cette intensité comme proportionnelle à la grandeur d'un effet produit par le courant (quantité d'un électrolyte décomposée pendant l'unité de temps, ou intensité d'un champ magnétique). Comme nous l'avons dit, ces définitions ne font intervenir aucune hypothèse sur la nature de courant et ne supposent pas définie la notion de quantité d'électricité. Avec cette manière de voir, la quantité d'électricité est une grandeur qui se déduit de l'intensité du courant. "

31 Ils présentent plus loin (p. 212) la théorie des ions mais n'emploient pas le terme électron:

"Des considérations sur lesquelles nous ne pouvons insister conduisent à penser que la structure de l'électricité est granulaire, comme celle de la matière, et la charge élémentaire dont nous venons de parler serait la plus petite quantité d'électricité qui puisse exister; cette charge serait indivisible et par suite toutes les autres en seraient des multiples entiers. "

On note le conditionnel prudent et dans l'édition de 1932, entièrement refondue de leur Nouveau cours de Physique élémentaire à destination des classes de Première, ces mêmes auteurs (Barrée remplaçant Brizard) ne mentionnent toujours pas l'électron, alors que pour la classe de philosophie dans une lecture (hors programme) sur « Les idées modernes sur la constitution de la matière »ils indiquent que l'hypothèse de la discontinuité de la matière «n'est pas absurde ».

Concernant les classes de mathématiques, il faut attendre l'édition de 1934 pour qu'à partir d'un paragraphe intitulé (p. 406) : «Idées modernes sur les charges électriques et sur le courant",ces auteurs utilisent les électrons pour interpréter un grand nombre de phénomènes.

"L'assimilation du courant électrique au déplacement d'un agent comparable à un fluide incompressible permet de supposer une circulation de charges positives dans le sens $d u$ courant, ou de charges négatives en sens inverse, ou les deux circulations à la fois. L'intensité du courant est la somme des valeurs absolues des charges qui traversent une section du circuit en une seconde. L'étude très étendue qui a été faite de l'Électricité, en particulier les recherches sur la conductibilité des électrolytes et sur la conductibilité des 
gaz, ont conduit à attribuer à l'électricité, comme à la matière, une structure discontinue, granulaire, et à considérer deux sortes de grains. Les charges négatives sont, dans les idées actuelles, constituées par des corpuscules plus ténus que l'atome d'hydrogène - le plus petit des atomes, cependant - tous identiques quelle que soit leur origine; ces corpuscules sont les électrons. Chacun d'eux porte la charge négative -e. Ils peuvent être libres, mais ils peuvent être également fixés à des atomes ou à des molécules neutres, formant ainsi des ions négatifs. Les charges positives sont toujours formées d'atomes qui ont perdu des électrons $[\ldots] »$ mathématiques spécialesaprès avoir présenté les théories anciennes de l'électricité éprouvent le besoin de préciser : «La théorie électronique s'appuie sur de nombreux faits démontrés et est universellement admise par tous les physiciens. Elle se rattache directement à la conception actuelle de la constitution de la matière [...]"

Il faut dire que le programme de Seconde de chimie précise explicitement en 1945 : "Théorie atomique ; symboles, formules, équations chimiques » et que pour les Terminales il est prévu une «Révision de la théorie atomique et de ses conséquences [...] ».

\subsection{En 1947}

A partir de 1947, les programmes de Terminales mentionnant en physique : «Émission électroniques (existence des effets thermoïoniques et photoélectrique; rayons cathodiques). Structure granulaire de l'électricité; négaton. [...] Idée d'une structure de l'atome [...]», les ouvrages se conformant à ces programmes, comme celui d'Eurin et Guimiot, consacrent un chapitre entier au négaton. On peut citer (p. 146): "Les rayons cathodiques sont constitués par des négatons, particules élémentaires d'électricité négative de charge -e» ou «Un courant électrique dans un conducteur est constitué par un courant de négatons circulant en sens opposé. » Dans l'ouvrage d'Ève, qui introduit en outre la structure du noyau, il est précisé que le terme négaton est employé à la place d'électron négatif car (p. 445): «On a récemment découvert l'existence de l'électron positif ou positon [...]».

Il est intéressant de noter que les instructions du 20 juin 1947 sur l'interprétation des programmes de sciences physiques des classes Terminales du second degré, reproduites dans l'ouvrage de Dumesnil et Lifermann, ne considèrent finalement ce programme que comme un programme de 1902 étoffé (p. 3) :

«En physique, avec quelques notions générales de dynamique et d'énergétique, nous retrouvons, comme en 1902, une étude assez complète, bien qu'élémentaire, des phénomènes périodiques s'étendant aux divers domaines: acoustique, optique et électricité. Quelques compléments relatifs aux émissions électroniques, aux substances radioactives, à la structure de l'atome donneront aux élèves une idée des récents progrès de la science [...]. L'exposé concernant le chapitre émissions électroniques sera très léger et réduit aux résultats essentiels; une expérience convenablement choisie mettra en évidence l'existence des effets thermoïoniques, une autre celle des effets photoélectriques; d'autres permettront d'observer les propriétés marquantes des rayons cathodiques et c'est dans leur interprétation que le maître sera conduit à parler de la structure granulaire de l'électricité et du négaton." 


\section{Commentaires}

On peut à travers ce panorama distinguer 4 périodes :

- jusque vers 1925, le terme électron n'apparait que de manière fortuite dans les ouvrages scolaires, en tant que charge élémentaire d'électricité, et les atomes ne sont considérés que comme des symboles facilitant l'écriture des réactions chimiques ;

- de 1925 à 1935, l'électron apparaît en physique comme un corpuscule et certains auteurs donnent une interprétation électronique de tous les phénomènes impliquant l'électron, alors que d'autres en parlent sans trop s'engager ; dans cette même période l'atome devient en chimie une hypothèse vraisemblable ;

- de 1935 à 1945, tous les ouvrages mentionnent l'électron, mais certains avec beaucoup plus de réserves que d'autres; de plus en plus d'ouvrages de chimie présentent la structure de l'atome;

- enfin après 1945, l'introduction de l'électron dans les programmes officiels impose d'en parler.

Toutefois certains ouvrages, par exemple le manuel de Chimie de Lamirand et Joyal à destination des élèves de Terminale, publié en 1951 (soit 6 ans après Hiroshima), et dans lequel un chapitre complet est consacré à présenter une structure assez détaillée de l'atome, emploient encore le mot hypothèse (p. 13) :

«La théorie atomique repose sur trois hypothèses fondamentales:

- 1. l'hypothèse moléculaire ;

- 2. l'hypothèse d'Avogadro ;

- 2. l'hypothèse atomique."

41 Dans ce cas, il ne semble pas y avoir tellement de différences avec le cours de chimie de Métrai de 1907. A moins qu'il s'agisse d'une confusion récurrente de formulation, volontaire ou non, avec le concept de modèle.

Pourtant au niveau de la recherche, L. Poincaré, indiquait dès 1908 : «[...] divers physiciens, M. Langevin par exemple, demandent que l'on fasse passer les atomes du rang des hypothèses à celui des principes ", au niveau de l'enseignement. Le même Langevin, cité par Hulin (1996), insiste déjà dès 1904 sur l'intérêt pédagogique de ces idées (p. 114) :

"Il suffit, d'ailleurs, de voir avec quelle avidité de bon aloi les élèves assimilent les quelques indications bien vagues que l'on peut se permettre à ce sujet [les idées atomistiques], où ils trouvent avec joie le support qui leur manque, pour être convaincus qu'on se prive volontairement dans l'enseignement secondaire d'un levier bien puissant, par un scrupule de rigueur tout à fait exagéré, et par suite du discrédit injuste dans lequel ces hypothèses sont tombées».

Aussi, est-ce vraiment un scrupule de rigueur qui explique que cette introduction de concepts de la physique moderne, notamment l'électron, s'est faite si lentement dans l'enseignement secondaire?

Nous écartons l'hypothèse d'un savoir non vraiment légitimé (tout au moins à partir de 1913) par la communauté scientifique.

Nous écartons aussi des arguments comme ceux avancés par $\mathrm{M}^{\text {lle }}$ Mourgues (1904), une des fondatrices de «l'Union Des Physiciens » (p. 56):

"Nos élèves n'ont que 18 ans. Est-il raisonnable de donner pour but à leurs recherches la constitution intime de la matière? N'y a-t-il pas un danger très grand à leur faire voir la vie 
au-delà de nos sens, même armés d'un puissant microscope ? Ont-ils assez de santé physique et morale pour supporter mieux que Pascal ce tête-à-tête avec l'Inconnu? "

\subsection{Ignorance du concept}

46 C'est l'explication la moins probable.

En 1934 est parue à la librairie Larousse, une sorte d'encyclopédie des sciences intitulée La Science, ses progrès, ses applications. Dans le tome second de cet ouvrage, au chapitre IV sur "Les Théories électroniques », l'auteur, M. Boll, parle de la déchéance du «fluide » électrique (p. 377) :

"Sur la foi de vulgarisateurs incompétents, qui retardent d'un quart de siècle, beaucoup d'esprit distingués répètent que 〈l'électricité est un fluide mystérieux». Cependant, dans le langage scientifique, le mot fluide n'est plus employé que pour désigner les liquides et le gaz, c'est-à-dire les substances qui n'ont pas de forme propre. Et, d'autre part, l'électricité n'est pas plus mystérieuse que la matière ou la chaleur; elle est, à coup sûr, bien moins mystérieuse que la lumière, pour laquelle ces mêmes esprit invoquent, constamment et à tort, l'éther, hypothèse périmée."

Alors qui sont ces vulgarisateurs incompétents? Ce ne sont pas des savants comme Boutaric qui à la même période fait paraître: La Physique Moderne et l'Électron, ou Millikan dont la traduction de la deuxième édition de son ouvrage L'Électron qui date de 1924, paraît en 1926.

Dès 1913, Guilleminot dans Les nouveaux horizons de la science introduit l'électron pour expliquer "Qu'est ce que l'électricité?", "Comment la notion d'électron explique le magnétisme de la matière » ou l'effet photoélectrique.

S'il est déjà peu plausible que ces auteurs n'aient pas été lus par les rédacteurs des manuels, il serait surprenant que La Physique Moderne, son évolution, due à un Inspecteur Général de l'Instruction Publique, L. Poincaré, ne l'ait pas été, d'autant plus que ce dernier a été membre de la sous-commission des sciences physiques pour la réforme des programmes de 1902. Or cet ouvrage date de 1908 et on y trouve entre autres (p. 242):

"L'idée que la conductibilité des métaux n'est pas essentiellement différente de celle des liquides électrolytiques ou des gaz, en ce sens que le passage du courant est lié au transport de petites particules électrisées, remonte déjà loin ; elle a été énoncée par Weber, développée ensuite par Giese, mais elle n'a vraiment pris son véritable sens qu'à la suite des découvertes récentes; ce sont les travaux de Riecke, puis de Drude, et surtout de J.J. Thomson, qui ont permis de lui donner une forme acceptable; tous ces essais se rattachent, d'ailleurs à la théorie générale de Lorentz, que l'on étudiera plus loin."

Il reprend les propos de Lodge de 1902 (p. 81): "Les métaux sont des corps tels que le transport d'un électron d'un atome à l'autre est facile et n'exige aucune force [...] ».

Autrement dit on ne peut invoquer la méconnaissance du concept d'autant plus que même les professeurs de base sont informés des développements de la science. En effet si l'on consulte le Bulletin de l'Union des Physiciens, créé en 1906 en vue de constituer pour les professeurs de sciences physiques un moyen d'être insérés dans un réseau d'échanges professionnels, dès les numéros de 1910 apparaissent des demandes de documentation relatives aux rayons $\mathrm{X}$, aux rayons cathodiques, à la radioactivité, aux travaux d'Einstein et de Planck. Une réponse parue dans le $\mathrm{N}^{\circ} 41$ de mars 1911 cite la traduction de l'ouvrage de Righi : La théorie moderne des phénomènes physiques : radioactivité, ions, électrons.

En 1915, M. Brillouin dans un article paru dans le $\mathrm{N}^{\circ} 83$ sur les «Mouvements de rotation. Quantité de mouvements » indique (p. 120) : "Tels sont les courants circulaires atomiques de la 
théorie d'Ampère et de Wéber ou les circulations de chaînes d'électrons qui les remplacent dans les formes plus modernes de cette théorie ".

Par la suite ce sont surtout les discussions sur l'introduction du potentiel et des polémiques extrêmement virulentes sur la relativité qui passionneront les lecteurs et auteurs des articles. L'électron s'est introduit naturellement. Et lorsque Déverin publie dans le numéro 118-119 de décembre 1918 - janvier 1919, un article «Sur la théorie des piles électriques» en faisant appel à la théorie des ions mais sans jamais parler d'électron, il provoque la remarque suivante d'un lecteur - qui signe simplement S. - publiée 6 mois plus tard (p. 151) : «[...] Et l'on peut se demander si le courant des électrons négatifs qui ferment le circuit dans le vide ne détrônera pas un jour la théorie des ions, ou du moins ne la modifiera pas profondément [...]».

54 Enfin dans une note de lecture sur L'électronde Millikan parue dans le № 197-198 de novembre-décembre 1926, celui qui a effectué la recension, Delvalez, commence ainsi (p. 77) : «J'ai eu l'occasion récemment de lire et de relire cet excellent livre; et je serais bien aise si les notes suivantes pouvaient en donner une idée convenable, et engager les professeurs à en faire l'acquisition pour eux-mêmes ou les bibliothèques des lycées et des collèges [...]».

\subsection{Inutilité du concept}

55 Les conseils généraux du programme de Seconde de 1902, auxquels il est fait référence pour les autres classes, sont explicites (p. 822):

"[...] Le but n'est pas de faire de nos élèves des physiciens de profession, mais de leur faire connaitre les grandes lois de la Nature et de les mettre à même de se rendre compte de ce qui se passe autour d'eux; dans cette vue l'enseignement doit être à la fois très élevé, très simple et très pratique. Évitant les développements mathématiques, il doit toujours être fondé sur des expériences [...]».

56 Ceci traduit d'une part, le positivisme des savants français de l'époque, et le clivage très fort qui existe alors entre les physiciens, expérimentateurs par définition, et les mathématiciens, catégorie qui englobe les physiciens théoriciens. En effet pour Comte, le mot positif désigne d'abord le réel par opposition au chimérique. Et les savants français comme Berthellot, qui attendent des expériences positives, vont privilégier le fait et la méthode expérimentale, par rapport aux hypothèses.

57 Outre ce positivisme fortement prégnant qui vise à rejeter tout modèle sous-jacent à la réalité observable, l'inductivisme est toujours la méthode officielle de présentation de la science. L'introduction des exercices pratiques dans la réforme de 1902 vise à initier l'élève à une démarche expérimentale, et renforce le caractère inductiviste de l'enseignement.

58 Ceci perdure longtemps puisque en 1947 les instructions officielles concernant la physique (Dumesnil \& Lifermann), précisent (p. 5) :

«Bien entendu, il n'est pas interdit au professeur d'employer à l'occasion, au lieu de la méthode inductive, la méthode déductive qui est particulièrement rapide, simple et claire et d'expliquer alors les faits à partir d'un principe posé à l'avance, mais à condition d'avoir montré aux élèves tout ce que ce procédé a d'artificiel du point de vue de l'édification de la Science. Nos jeunes gens ne doivent pas oublier que la Science s'est faite lentement au cours des siècles, que sa construction a exigé des efforts multiples, qu'elle s'est accompagnée de bien des tâtonnements et a subi de nombreux échecs avant d'atteindre sa forme actuelle qui ne saurait être définitive. » 
59 même auteur, qui concernent la Chimie en classe Terminale, publiées le même jour (20 juin 1947) et reproduites dans l'ouvrage de Lamirand et Joyal (p. 3) :

"[...] Dans son exposé, le professeur abandonnera délibérément la méthode historique de présentation et prendra pour base la théorie atomique qu'il esquissera à grands traits, se réservant de revenir en fin d'année, après l'étude des rayonnements et des substances radioactives, sur la constitution de l'édifice atomique. Ce procédé est peut-être dogmatique, mais il est particulièrement simple, rapide et il donne aux lois générales (Lavoisier, Dalton, Proust... ) un caractère d'évidence qui fait que les élèves les retiennent sans aucune peine.»"

Il a donc fallu 50 ans pour que l'électron apparaisse dans les programmes officiels car le caractère expérimental sans cesse réaffirmé, et encore jusqu'à aujourd'hui, des sciences physiques renforçait la notion de vérité positive, c'est-à-dire celle du fait expérimentalement constaté. Or à quoi bon parler de la nature de l'électricité si l'on est capable de rendre compte par des lois simples des mesures effectuées, ou mieux encore si, à partir des mesures, on est capable d'induire des lois explicatives.

Figure 6

" Euf philosophique : ballon dans lequel on peut faire le vide, et qui porte deux conducteurs électriques. Quand le vide est fait, l'électricité passe non pas sous forme d'étincelle, mais sous la forme d'un grand globe de feu qui remplit le ballon. À mesure qu'on laisse rentrer l'air, ce globe se resserre, il se transforme en nappes, en sillons, en filaments ; enfin, à une certaine pression, l'électricité ne passe plus. "

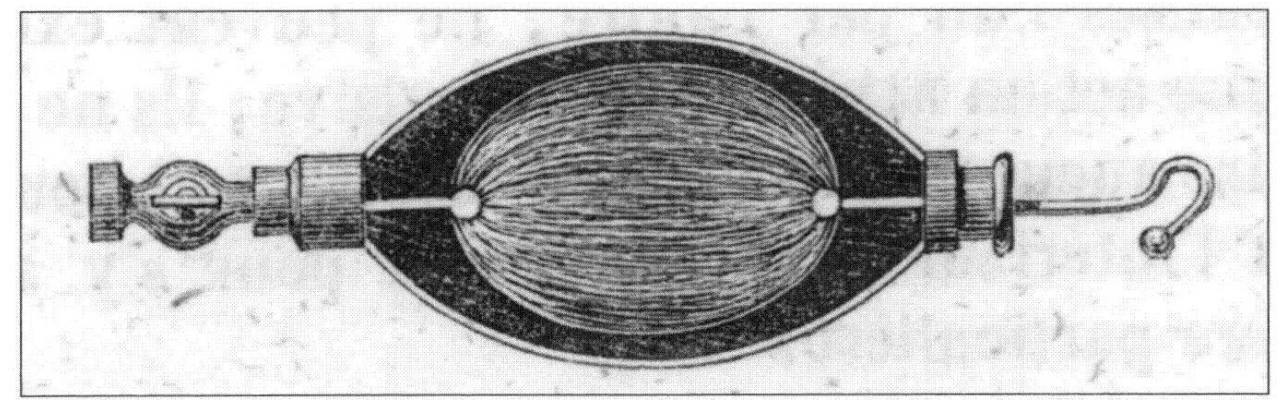

POUILLET M. :Notions Générales de Physique et de Météorologie à l'Usage de la Jeunesse. Paris, Béchet Jeune, 1850, p. 280.

61 Une fois le barrage antiatomisme rompu, le concept d'électron s'est imposé en chimie car il permettait d'expliquer de manière simple et auto-cohérente de plus en plus de propriétés dues au caractère électronique de la liaison chimique. La logique déductive s'imposait sur l'inductivisme par sa facilité pédagogique d'exposition.

\section{Conclusion}

Avec l'électron, nous nous trouvons en présence d'un exemple bien particulier d'un savoir qui se développe dans la communauté scientifique tout en bouleversant la technologie, notamment à travers l'électronique naissante, sans que cela ait de réelles répercussions sur l'enseignement secondaire.

$63 \mathrm{Au}$ delà des explications fournies précédemment, on peut également interpréter le décalage observé en considérant que les contenus d'enseignement sont aussi le fruit d'une demande sociale. 
64 En 1902 les finalités de l'enseignement secondaire sont d'ordre culturel, comme le précisent les conseils généraux des programmes de physique-chimie, que nous avons rappelés au début du paragraphe 2.2 , et dans cette réalité observable, on ne "voit » pas les électrons. Ces humanités scientifiques se veulent paradoxalement à la fois désintéressées et extrêmement concrètes.

Après la seconde guerre mondiale, l'importance toujours croissante de la science et de la technologie dans la société se traduit d'une part par une forte demande en ingénieurs et en techniciens, et d'autre part corrélativement, dans une frange de plus en plus importante de la population, par le besoin d'une plus grande intelligibilité du monde qui nous entoure. Cette double exigence impose à l'enseignement des sciences physiques de coller au plus près à la production de connaissances scientifiques en allant au delà de la simple mise en relation des faits.

\section{BIBLIOGRAPHIE}

BOLL M. : « Les théories électroniques ", in La Science, ses progrès, ses applications. Tome 2 : Les applications et les théories actuelles (Dir. : URBAIN G. \& Boll M.). Paris, Larousse, 1934.

BOUASSE H. \& BRIZARD L. : Physique (Classe de première). Paris, Delagrave, 1925.

BOUTARIC M.A. : La Physique moderne et l'Électron. Paris, Éd. : F. ALCAN, 1935.

BRUHAT G. : Cours d'électricité à l'usage de l'enseignement supérieur scientifique et technique. Paris, Masson, 1924.

DAGUIN P.A. : Traité élémentaire de Physique théorique et expérimentale avec les applications à la météorologie et aux arts industriels. Paris, Delagrave, 1867.

DRINCOURT E. : Trois années de Chimie (en un volume). Paris, A. Colin, 1909.

DUMESNIL G. \& LIFERMANN J. : Physique (Classes de mathématiques élémentaires et Sciences Expérimentales). Paris, ISTRA, 1948.

DUMAS J.-B. : Leçons sur la philosophie chimique. Paris ; Bruxelles, Éditions Culture et civilisation, 1837, (Ré-édition : 1972).

EURIN M. \& GUIMIOT H. : Sciences Physiques (classe de philosophie). Paris, Hachette, 1947.

ÈVE G. : Physique (Classes de mathématiques et Sciences Expérimentales). Paris, Magnard, 1947.

FAIVRE-DUPAIGRE J. \& CARIMEY E. : Nouveau cours de Physique élémentaire (classe de Première). Paris, Masson, 1913.

FAIVRE-DUPAIGRE J., LAMIRAND J. \& BRIZARD L. : Nouveau cours de Physique élémentaire (classe de Première). Paris, Masson, 1932.

FAIVRE-DUPAIGRE J., LAMIRAND J. \& BARRÉE M. : Cours de Physique pour les classes de mathématiques spéciales. Paris, Masson, 1932.

FAIVRE-DUPAIGRE J., LAMIRAND J. \& BARRÉE M. : Nouveau cours de Physique élémentaire (classe de mathématiques). Paris, Masson, 1934. 
FAIVRE-DUPAIGRE J., LAMIRAND J. \& JOYAL M. : Cours de Physique pour les classes de mathématiques spéciales; Tome III : Électricité. Paris, Masson, 1946.

FERNET E. (en collaboration avec FAIVRE-DUPAIGRE J.) : Précis de Physique. Paris, Masson, 1905. FRÉCHENGUES P. \& DUSSEAU J.-M. : « Bref historique de l'émergence du concept d'électron », in Bulletin de l'Union des Physiciens. Paris, Vol. 92, N 808, 1998.

GUILLEMINOT H. : Les nouveaux horizons de la science, Tome deuxième : l'électricité, les radiations, l'éther, origine et fin de la matière. Paris, G. Steinheil, 1913.

HULIN N. : « Constitution de la Physique moderne », in Les sciences au lycée (Dir. : BELHOSTE B., G ISPERT H. \& HULIN N.). Paris, Vuibert et INRP, 1996, pp. 55-68.

LAMIRAND J. \& JOYAL M. : Chimie (Classes de mathématiques et Sciences Expérimentales). Paris, Masson, 1951.

LELONG B. : « Paul Villard, J.J. Thompson et la composition des rayons cathodiques », in Revue d'Histoire des Sciences, Tome 50, Nº 1-2, pp. 91-130. Paris, PUF, 1997.

LEMOINE J. \& VINCENT G. : Cours élémentaire de Physique (Classe de mathématiques). Paris, E. Belin, 1908, 1925.

LEMOINE J. \& VINCENT G. : Cours élémentaire de Physique (Classe de Première). Paris, E. Belin, 1925.

LEMOINE J. \& VINCENT G. : Cours élémentaire de Physique (Classe de mathématiques). Paris, E. Belin, 1931.

LODGE O. : Sur les électrons. Conférence faite à l'« Institution of Electrical Engineers » le 5/11/1902. Paris, Gauthier-Villars, 1906.

MÉTRAL P. : Cours de Chimie. Paris, Masson, 1907.

MÉTRAL P. : Cours de Physique pour l'Enseignement Primaire Supérieur. Paris, Masson, 1907.

MÉTRAL P : Cours de Physique pour les Écoles primaires supérieures de garçons. Paris, Masson, 1909.

MOURGUES : in Langevin, (Dir. : BENSAUdE-VINCENT B., 1987). Paris, E. Belin, 1904, p. 56.

MILLIKAN R.A. : L'électron. Paris, Alcan, 1926.

OLLIVIER H. : Cours de Physique générale à l'usage des élèves des facultés des sciences. Paris, Hermann, 1932.

PASTOURIAUX L. \& COINTET A. : Chimie, $2^{e}$ année d'École Normale. Paris, Delagrave, 1925.

POINCARÉ L. : La Physique moderne, son évolution. Paris, E. Flammarion, 1908.

PÉCHARD E. : Chimie élémentaire PCN. Paris, Gautier-Villars, 1926.

PÉCHEUX H. : Précis de Physique industrielle. Paris, J.-B. Baillière, 1899.

PLANTÉ G. : Recherches sur l'Électricité. Paris, La lumière Électrique, 1883.

PONSINET J. : Principes de l'électrochimie. Paris, A. Colin, 1927.

POUILLET M. : Notions générales de Physique et de météorologie à l'usage de la jeunesse. Paris, Béchet Jeune, 1850.

RAMBOSSON M.J. : La science populaire ou revue du progrès des connaissances et de leurs applications aux arts et à l'industrie. Paris, E. Lacroix, 1863. 
TURPAIN A. : Physique à l'usage des élèves des classes de Seconde et Première $C$ et $D$ et Mathématiques A et B. Paris, Vuibert, 1927.

WURTZ A. : La théorie atomique. $2^{\mathrm{e}}$ édition, Paris, Alcan, 1879.

\section{RÉSUMÉS}

Non disponible

Not available

INDEX

Mots-clés : électron, enseignement secondaire, France, inductivisme, manuel scolaire, positivisme, sciences physiques

Keywords : inductivism, physics, positivism, school textbook, secondary school teaching

\section{AUTEURS}

\section{JEAN-MICHEL DUSSEAU}

Laboratoire ERES de l'université de Montpellier II, groupe IUFM

PIERRE FRÉCHENGUES

Laboratoire ERES de l'université de Montpellier II, groupe IUFM 